\title{
Titrimetric Study of the Solubility and Dissociation of Benzoic Acid in Water: Effect of Ionic Strength and Temperature
}

\author{
Sa'ib J. Khouri \\ Department of Basic Sciences, American University of Madaba, Madaba, Jordan \\ Email: sbkhouri@yahoo.com, s.khouri@aum.edu.jo
}

Received 7 March 2015; accepted 1 April 2015; published 3 April 2015

Copyright (C) 2015 by author and Scientific Research Publishing Inc.

This work is licensed under the Creative Commons Attribution International License (CC BY).

http://creativecommons.org/licenses/by/4.0/

(c) (7) Open Access

\begin{abstract}
The apparent acid dissociation constant $(K c)$ of benzoic acid in water has been determined titrimetrically under ionic strength values between 0.00 and $0.50 \mathrm{~mol} \cdot \mathrm{L}^{-1}$ at a range of temperatures between $16^{\circ} \mathrm{C}$ and $41^{\circ} \mathrm{C}$. The thermodynamic dissociation constant (as $\mathrm{pKa}$ ) of benzoic acid was determined as 4.176 at $25^{\circ} \mathrm{C}$. No regular correlation between $p K a$ of benzoic acid and the temperature in the range was used. The values of $p K a$ are inversely proportional to temperatures between $16^{\circ} \mathrm{C}$ and $30^{\circ} \mathrm{C}$. In this range of temperature, the values of thermodynamic quantities $\left(\Delta H^{\circ}\right.$, $\Delta S^{o}$ and $\Delta G^{o}$ ) for the dissociation process of benzoic acid in water were calculated by using Van't Hoff plot. For this case the dissociation was not favoured through entropy and enthalpy changes. The values of $\mathrm{pKa}$ are directly proportional to temperatures between $30^{\circ} \mathrm{C}$ and $41^{\circ} \mathrm{C}$.
\end{abstract}

\section{Keywords}

Benzoic Acid, Ionic Strength, Thermodynamic Dissociation Constant, Salt Effect

\section{Introduction}

Equilibrium studies concerning the solubility and the dissociation process of many well-known weak acids in aqueous solutions have been reported over the years by following many physical and analytical methods. Benzoic acid, $\mathrm{C}_{6} \mathrm{H}_{5} \mathrm{COOH}$, is one of the simplest organic acids of aromatic series with low solubility in water. Its high water soluble sodium salt (sodium benzoate) is used as food preservative to inhibit the growth of yeasts and moulds. Benzoic acid dissociates partially in water to produce benzoate anion and hydronium cation with an apparent dissociation constant that depends on many factors. It is well-known that the temperature and ionic 
strength can affect the equilibrium state of weak acids in solution, which is of great importance in chemical and biomedical analysis [1] [2]. Water molecules can attach to benzoic acid by hydrogen bonding and work to stabilizing the formation of the "benzoate" ion. The addition of salts in water gives rise to slight changes in the water-water interactions and that also affects the solute-solute correlations [3]. Benzoic acid dissolves only slightly in cold water though the polarization of carboxylic acid group. The bulk of the benzoic acid molecule has no internal stabilizing structure that favours carboxylate group over carboxylic acid that makes the molecule behave as a weak electrolyte in aqueous solutions [4].

In this work, the apparent dissociation constants $(K c)$ of benzoic acid in aqueous solutions have been determined titrimetrically with different values of ionic strengths at a small range of temperatures. For each case the thermodynamic dissociation constant (as $p K a$ ) has been evaluated graphically by using equations derived from Debye-Hückel Limiting law, which was improved and used in this work to obtain more accurate results as compared to the reported $p K a$ values in the literature. The thermodynamic parameters of the dissociation process of benzoic acid in water have been reported at standard condition. The absence of such information on benzoic acid in the literature following this method motivated us to carry out this study.

\section{Theory}

In a saturated aqueous solution, benzoic acid has a little molar solubility with the following equilibrium:

$$
\mathrm{C}_{6} \mathrm{H}_{5} \mathrm{COOH}_{(\mathrm{aq})} \stackrel{K c}{\rightleftarrows} \mathrm{H}_{(\text {aq) }}^{+}+\mathrm{C}_{6} \mathrm{H}_{5} \mathrm{COO}_{(\text {aq) }}^{-}
$$

where $K c$ is the apparent dissociation constant of benzoic acid, which is affected by several factors including temperature and ionic strength. The total molar solubility of benzoic acid in water is

$$
\begin{array}{r}
{\left[\mathrm{C}_{6} \mathrm{H}_{5} \mathrm{COOH}_{(\text {aq) }}\right]+\left[\mathrm{C}_{6} \mathrm{H}_{5} \mathrm{COO}^{-}\right]} \\
\text {or } y=\left[\mathrm{C}_{6} \mathrm{H}_{5} \mathrm{COOH}_{(\mathrm{aq})}\right]+x
\end{array}
$$

where $y$ represents the total molar solubility and $x$ represents the molar solubility of benzoate ion or hydrogen ion in aqueous solution,

$$
x=\left[\mathrm{C}_{6} \mathrm{H}_{5} \mathrm{COO}^{-}\right]=\left[\mathrm{H}^{+}\right]=10^{-\mathrm{pH}}
$$

Total molar solubility can be determined titrimetrically against a standardized strong base solution.

The equilibrium in Equation (1) can be expressed by two ways

$$
\begin{gathered}
K c=\frac{\left[\mathrm{H}^{+}\right]\left[\mathrm{C}_{6} \mathrm{H}_{5} \mathrm{COO}^{-}\right]}{\left[\mathrm{C}_{6} \mathrm{H}_{5} \mathrm{COOH}_{(\mathrm{aq})}\right]} \\
K a=\frac{\left[\mathrm{H}^{+}\right]\left[\mathrm{C}_{6} \mathrm{H}_{5} \mathrm{COO}^{-}\right]}{\left[\mathrm{C}_{6} \mathrm{H}_{5} \mathrm{COOH}_{(\mathrm{aq})}\right]} \cdot \frac{\gamma_{\gamma} \gamma_{-}}{\gamma_{u}}
\end{gathered}
$$

where $\gamma_{+}$and $\gamma_{-}$are activity coefficients of the dissociated ions of benzoic acid and $\gamma_{u}$ is that for undissociated benzoic acid; $\mathrm{Ka}$ is the thermodynamic dissociation constant at infinite dilution at a given temperature [5].

From Equations (4) and (5)

$$
K a=K c \cdot \frac{\gamma_{+} \gamma_{-}}{\gamma_{u}}
$$

$\gamma_{+} \gamma_{-}=\gamma_{ \pm}^{2}$, where $\gamma_{ \pm}$is the mean activity coefficient of the dissociated ions and is affected by ionic strength of the solution, while $\gamma_{u}=1$ is for undissociated benzoic acid in solution.

$$
I=\frac{1}{2} \sum_{i} c_{i} z_{i}^{2}
$$

where $I$ is the ionic strength; $c_{i}$ is the molar concentration of ion of $i$ th type; and $z_{i}$ is its charge number of $i$ th 
type in solution. In this work the contribution of benzoic acid to ionic strength $(I)$ values is ignored due to its relatively small dissociation. Sodium chloride is used in this work as an electrolyte to raise the ionic strength in each of particular solution of benzoic acid and the value of ionic strength $(I)$ for each solution is the molar concentration of $\mathrm{NaCl}$.

$\gamma_{ \pm}$is related to ionic strength $(I)$ by the following law:

$$
\log \gamma_{ \pm}=-B\left|z_{+} z_{-}\right| \sqrt{I}
$$

where $B$ is a quantity that depends on some physical properties of the solution such as dielectric constant and temperature [5].

Equation (6) and Equation (8) give

$$
\begin{gathered}
K a=K c \gamma_{ \pm}^{2} \\
\log K a+2 B \sqrt{I}=\log K c
\end{gathered}
$$

For aqueous solutions at $25^{\circ} \mathrm{C}$, Equation (10) becomes

$$
\log K a+1.02 \sqrt{I}=\log K c
$$

\section{Experimental}

All chemicals used in this study were of analytical reagent grade and were used without further purification. The solvent used throughout was water, which was three times distilled. Carbonate-free $\mathrm{NaOH}$ solutions were prepared as suggested by Vogel [6].

In a typical preparation for the solutions, $1 \mathrm{~g}$ of benzoic acid was placed in each of six dry $250 \mathrm{ml}$ stoppered bottles. In each bottle, $100 \mathrm{ml} \mathrm{NaCl}$ solution prepared by $100 \mathrm{ml}$ volumetric flask of concentrations $0.00,0.050$, $0.10,0.30,0.40$, and $0.50 \mathrm{M}$ were poured in these bottles and then put in a thermostat at specific temperature with shaking vigorously in the beginning, and then occasionally for two hours. From each bottle $20.0 \mathrm{ml}$ was withdrawn by $20.0 \mathrm{ml}$ pipette with filter to prevent drawing small solids into the pipette, then discharged to 250 $\mathrm{ml}$ conical flask after removing the filter. The concentration of benzoic acid in each solution was determined by titration with $0.050 \mathrm{M} \mathrm{NaOH}$. The $\mathrm{pH}$ of each solution in the bottles was measured by using 3 decimal digits calibrated $\mathrm{pH}$-meter at the different temperatures used. Class " $\mathrm{A}$ " calibrated volumetric glassware were used in this work.

\section{Results and Discussion}

\subsection{Effect of Salts on the Solubility and the Apparent Dissociation Constant at $25^{\circ} \mathrm{C}$}

The influence of the ionic strength on the solubility and the apparent dissociation constant $(K c)$ of benzoic acid at $25^{\circ} \mathrm{C}$ was examined at six different ionic strengths in the range of $0.0013-0.50 \mathrm{M}$ by adding $\mathrm{NaCl}$. The solubility of benzoic acid in water for each concentration of $\mathrm{NaCl}$ was evaluated titrimetrically, followed by measuring the $\mathrm{pH}$ of each solution. The experimental data are presented in Table 1, which taken as average of several results of measurements that show high precision. The observed solubility (from the amount of sodium hydrox-

Table 1. Effect of $\mathrm{NaCl}$ concentration on the solubility of benzoic acid in water at $25^{\circ} \mathrm{C}$.

\begin{tabular}{cccc}
\hline$[\mathrm{NaCl}] \mathrm{mol} / \mathrm{L}$ & Volume of benzoic acid solution $/ \mathrm{mL}$ & Volume of $0.050 \mathrm{~mol} / \mathrm{L} \mathrm{NaOH} / \mathrm{mL}$ & $\mathrm{pH}$ \\
\hline 0.000 & 20.0 & $10.61 \pm 0.014$ & $2.884 \pm 0.005$ \\
0.050 & 20.0 & $10.19 \pm 0.010$ & $2.832 \pm 0.005$ \\
0.10 & 20.0 & $9.92 \pm 0.009$ & $2.813 \pm 0.003$ \\
0.30 & 20.0 & $9.51 \pm 0.012$ & $2.789 \pm 0.005$ \\
0.40 & 20.0 & $9.09 \pm 0.011$ & $2.763 \pm 0.003$ \\
0.50 & 20.0 & $8.58 \pm 0.011$ & $2.747 \pm 0.007$ \\
\hline
\end{tabular}


ide solution) and $\mathrm{pH}$ values of benzoic acid in water related inversely with $\mathrm{NaCl}$ concentrations. For each solution, the apparent dissociation constant $(K c)$ of benzoic acid was evaluated by using the following modulated expression that derived from Equation (4) and from the expression of finding $\mathrm{pH}$ value:

$$
K c=\frac{\left(10^{-\mathrm{pH}}\right)^{2}}{\left(y-10^{-\mathrm{pH}}\right)}
$$

where $y$ is the total molar solubility of benzoic acid in solution $\left(\left[\mathrm{C}_{6} \mathrm{H}_{5} \mathrm{COOH}_{(\mathrm{aq})}\right]+\left[\mathrm{C}_{6} \mathrm{H}_{5} \mathrm{COO}^{-}\right]\right)$that could be evaluated from the following expression:

$$
y=\left(V_{\mathrm{NaOH}} \times M_{\mathrm{NaOH}}\right) / V_{\mathrm{BA}}
$$

where $V_{\mathrm{NaOH}}, M_{\mathrm{NaOH}}$, and $V_{\mathrm{BA}}$ are respectively; volume of sodium hydroxide per liter, molarity of sodium hydroxide per mol/L, and volume of benzoic acid sample per liter. Table 2 contains values of $y, 10^{-\mathrm{pH}}, \mathrm{Kc}$, and ionic strength for each benzoic acid solution at $25^{\circ} \mathrm{C}$. Figure 1 shows the plot of $(\log K c)$ as the y-axis versus $\sqrt{I}$ as the $\mathrm{x}$-axis, which illustrates direct and linear relationship after applying the linear least-squares analysis of the data $\left(r^{2}=0.99188\right)$. The value of the actual thermodynamic dissociation constant, $\mathrm{Ka}$, of benzoic acid at $25^{\circ} \mathrm{C}$ can be obtained by extrapolation to zero ionic strength. The value of estimated logKa from the plot is $-4.176 \pm 0.01035$, which its inverse (4.176) represents $p K a$ of benzoic acid at $25^{\circ} \mathrm{C}$. This value is in a good agreement with reported values in the literature from using different methods; electrophoresis 4.12 [7], potentiometry 4.20 [8], spectrometry 4.19 [9], and liquid chromatography 4.18 [10].

The mean activity coefficient of the dissociated ions $\left(\gamma_{ \pm}\right)$could be calculated for each solution from the following equation that derived from Equation (9):

$$
\log \gamma_{ \pm}=1 / 2(\log K a-\log K c)
$$

Plotting $\log \gamma_{ \pm}$as the $\mathrm{y}$-axis versus $\sqrt{I}$ as the $\mathrm{x}$-axis (Figure 2) gives rise to a straight line with a limiting slope equal to -0.2698 . The value of mean activity coefficient for any concentration of sodium chloride in the range used can be estimated from this plot.

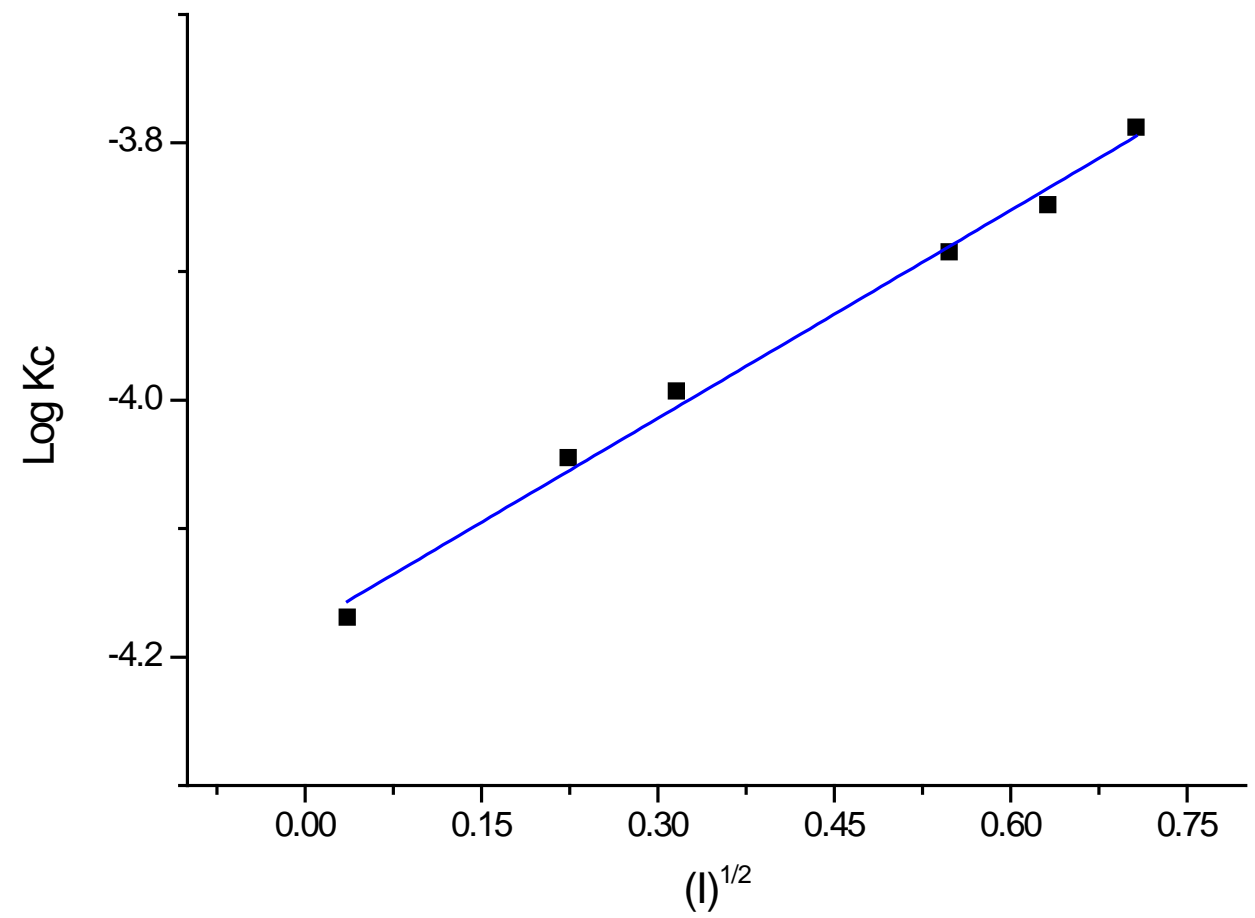

Figure 1. The effect of ionic strength on the values of acid dissociation constant $(K c)$ of benzoic acid in water at $25^{\circ} \mathrm{C}$. 


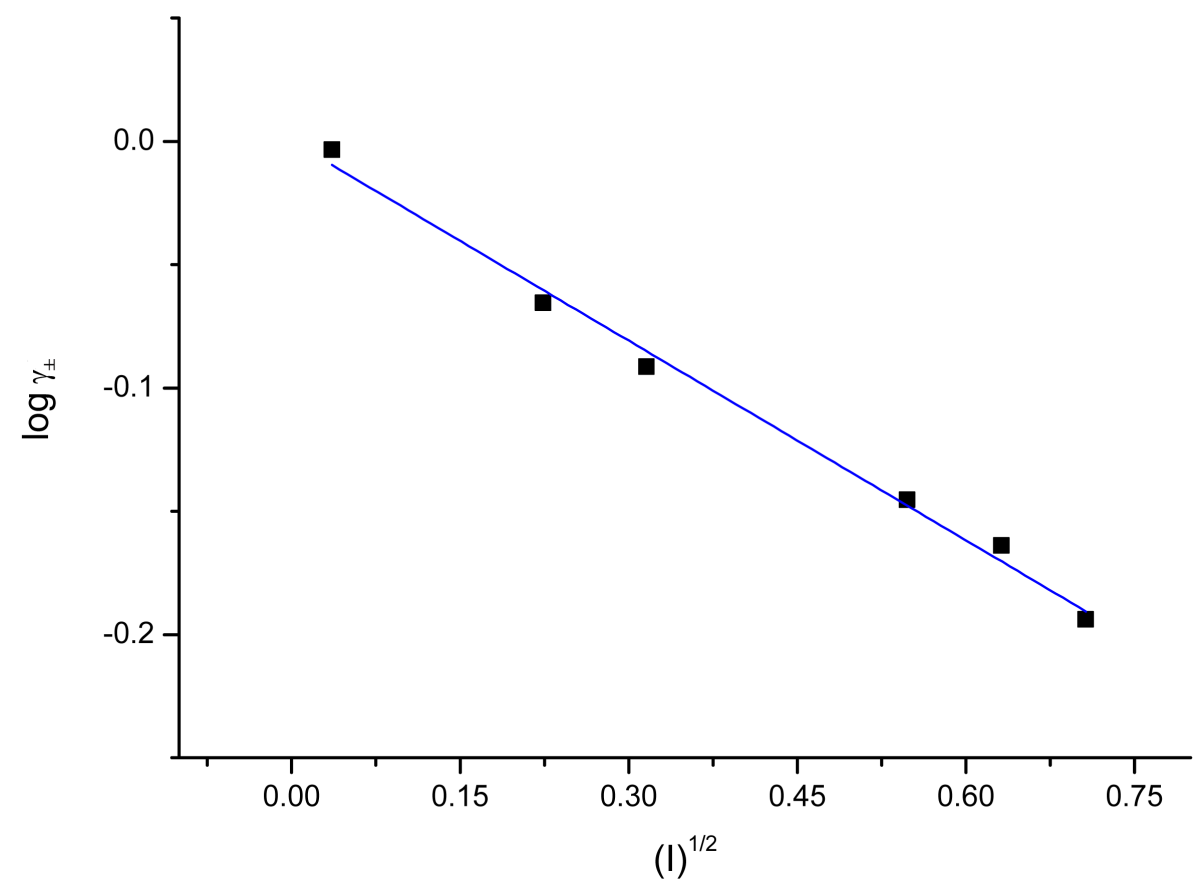

Figure 2. The effect of ionic strength on the values of the mean activity coefficient $\left(\gamma_{ \pm}\right)$of benzoic acid in water at $25^{\circ} \mathrm{C}$.

Table 2. Effect of ionic strength on the value of acid dissociation constant of benzoic acid in water at $25^{\circ} \mathrm{C}$.

\begin{tabular}{|c|c|c|c|c|c|}
\hline Ionic strength, I/m & $y$ & $10^{-\mathrm{pH}} 10^{-3}$ & $\frac{\left(10^{-\mathrm{pH}}\right)^{2}}{\left(y-10^{-\mathrm{pH}}\right)} 10^{-4}$ & $\log \frac{\left(10^{-\mathrm{pH}}\right)^{2}}{\left(y-10^{-\mathrm{pH}}\right)}$ & $\sqrt{I}$ \\
\hline 0.00 & 0.0265 & 1.306 & 0.6770 & -4.169 & 0.036 \\
\hline 0.05 & 0.0255 & 1.472 & 0.9018 & -4.045 & 0.224 \\
\hline 0.10 & 0.0248 & 1.538 & 1.017 & -3.993 & 0.316 \\
\hline 0.30 & 0.0238 & 1.626 & 1.192 & -3.885 & 0.548 \\
\hline 0.40 & 0.0227 & 1.726 & 1.420 & -3.848 & 0.632 \\
\hline 0.50 & 0.0215 & 1.791 & 1.628 & -3.788 & 0.707 \\
\hline
\end{tabular}

\subsection{Effect of Temperature on the Equilibrium Dissociation Constant}

By repeating the same procedure (as in the last section) six more times with temperature values around $25^{\circ} \mathrm{C}$, the solubility of benzoic acid in water for the same concentrations of $\mathrm{NaCl}$ used at $25^{\circ} \mathrm{C}$, was evaluated titrimetrically at temperatures between $16^{\circ} \mathrm{C}$ and $41^{\circ} \mathrm{C}$, followed by measuring the $\mathrm{pH}$ of each solution (the average of the results that show high precision at each temperature was taken for $\mathrm{NaOH}$ volume and $\mathrm{pH}$ values). At each temperature, $K c$ value for each $\mathrm{NaCl}$ concentration used was calculated by the same method as done in the case of $25^{\circ} \mathrm{C}$, and the thermodynamic dissociation constant (as $p K a$ ) was also estimated from plots of logKc vs $\sqrt{I}$ for each temperature. In thermodynamics the use of molarity is often not convenient, because the volume of most solutions slightly depends on temperature due to thermal expansion. This problem was resolved by introducing temperature correction to maintain the same $\mathrm{NaCl}$ concentration for all temperatures used, such correction was worked by testing the increase or decrease in solution volume of $\mathrm{NaCl}$ inside the $100 \mathrm{~mL}$ volumetric flask at each selected temperature relative to the standard volume of the flask at $20^{\circ} \mathrm{C}$, then preparing the $\mathrm{NaCl}$ solution with a starting volume more or less than $100 \mathrm{~mL}$ to be exactly $100 \mathrm{~mL}$ when attained thermal equilibrium inside the thermostat with the selected temperature. Figure 3 illustrates the relation between the estimated $p K a$ 


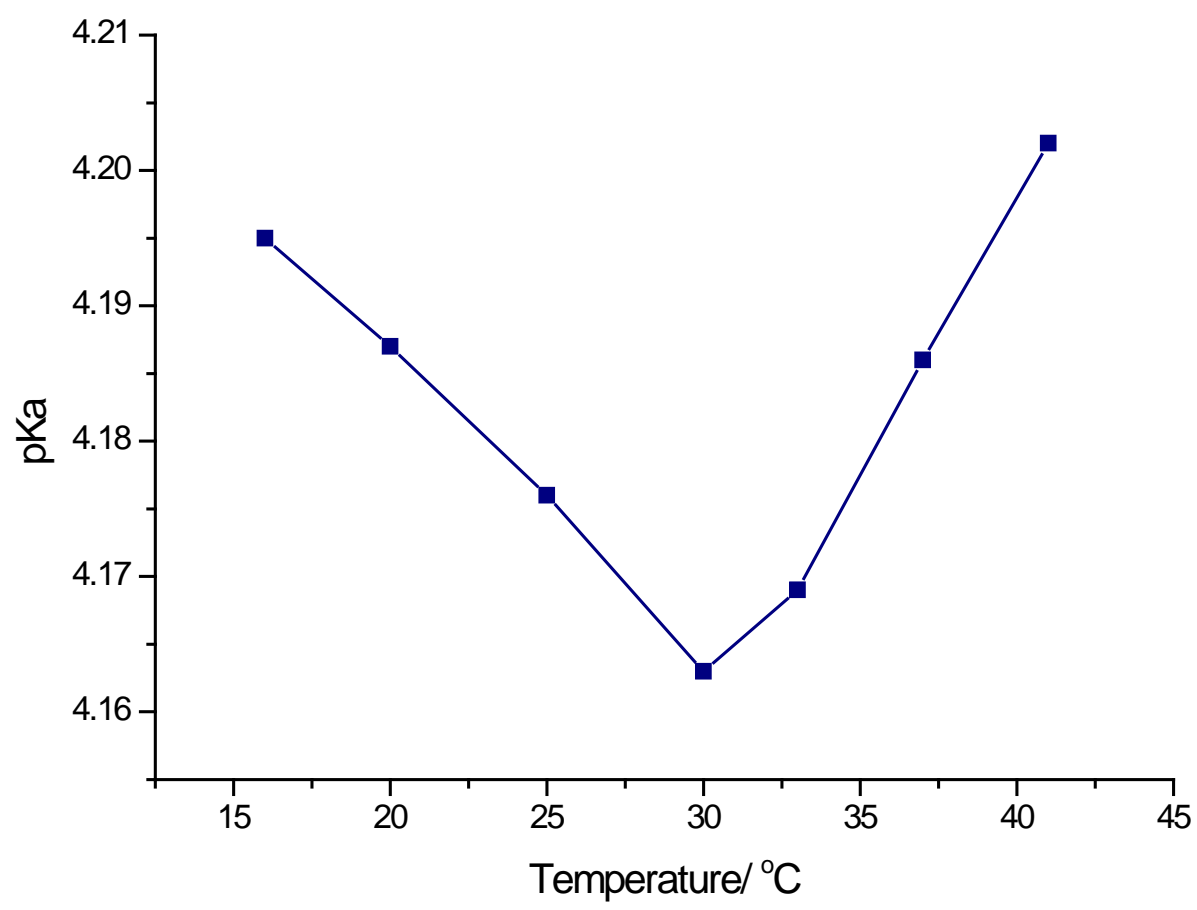

Figure 3. The effect of temperature on the value of the thermodynamic dissociation constant $(K a)$ of benzoic acid in water.

values and temperatures used. In the Figure, the values of $p K a$ are inversely proportional with temperature between $16^{\circ} \mathrm{C}$ and $30^{\circ} \mathrm{C}$. In contrast the values of $p K a$ are directly proportional with temperature between $30^{\circ} \mathrm{C}$ and $41^{\circ} \mathrm{C}$, i.e. no regular correlation between $\mathrm{pKa}$ of benzoic acid and range of temperatures that used. From the experimental data; the amount of $\mathrm{NaOH}$ used in titration and the value of $\mathrm{pH}$ for each temperature used, the solubility of benzoic acid in water is directly proportional with temperature, and the capability of the benzoic acid molecule to dissociate is not always increases as temperature increases.

The values of enthalpy change $\left(\Delta H^{\circ}\right)$ and entropy change $\left(\Delta S^{\circ}\right)$ of the dissociation process of benzoic acid in the ordinary temperature range between $16^{\circ} \mathrm{C}$ and $30^{\circ} \mathrm{C}$ were obtained according to the following linear thermodynamic equation (Van’t Hoff equation):

$$
\log K a=\frac{-\Delta H}{2.303 R} \frac{1}{T}+\frac{\Delta S}{R}
$$

where $R$ is the molar gas constant $\left(8.314 \mathrm{~J} \cdot \mathrm{K}^{-1} \cdot \mathrm{mol}^{-1}\right)$, and taken into account that $\Delta H^{o}$ and $\Delta S^{o}$ are independent on temperature due to the relatively small change in temperature values. A plot of $\log (K a)$ as $y$-axis against $1 / T$ as $\mathrm{x}$-axis gives $\Delta H^{o}$ and $\Delta S^{o}$ values. Gibbs free energy $\left(\Delta G^{o}\right)$ was calculated from the following thermodynamic relation:

$$
\Delta G^{o}=\Delta H^{o}-T \Delta S^{\circ}
$$

Figure 4 shows the plot of Equation (15) for benzoic acid in water at the temperature range between $16^{\circ} \mathrm{C}$ and $30^{\circ} \mathrm{C}$. A linear least-squares analysis of $\log K a$ vs. $1 / T$ data illustrates a linear relationship $\left(r^{2}=0.99237\right)$ and gave the following results for the standard values of the thermodynamic parameters for the dissociation of benzoic acid in water: $\Delta H^{o}=3.823 \mathrm{~kJ} / \mathrm{mol}, \Delta S^{o}=-29.14 \mathrm{~kJ} / \mathrm{mol}$, and $\Delta G^{o}=12.507 \mathrm{~kJ} / \mathrm{mol}$.

Although the details of a dissociation process are not usually revealed by a thermodynamic study, a conclusion can be drawn from the above results that the dissociation process represented by Equation (1) is nonspontaneous and endothermic. It is clear that both the enthalpy change and the entropy change make benzoic acid molecule to be not favoured to dissociate in water between $16^{\circ} \mathrm{C}$ and $30^{\circ} \mathrm{C}$. These two thermodynamic parameters; positive enthalpy change and negative entropy change contribute in retarding the dissociation process making Gibbs energy change to be positive, therefore the dissociation process of benzoic acid molecule is non- 


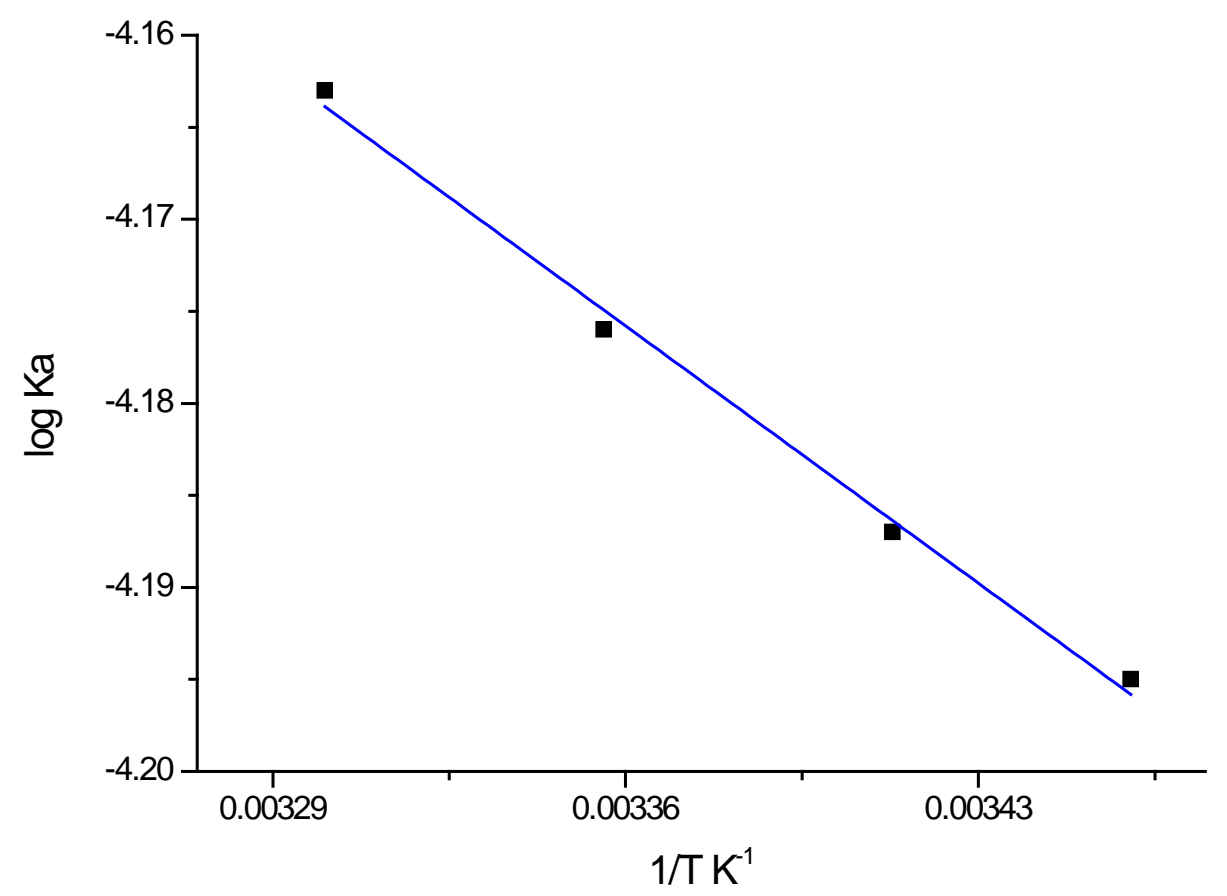

Figure 4. Plot of Equation (15) for benzoic acid in water.

spontaneous. Moreover, the negative entropy change means that benzoic acid in water attain a more ordered state after its dissociation process.

At a temperature higher than $30^{\circ} \mathrm{C}$, benzoic acid in water behaves differently. As a temperature increases the capability of benzoic acid to dissociate decreases and that leads to reduce the value of $K a$. The process in this range is certainly exothermic and compatible with Le Chatelier's principle [5]. The effect of temperature on the acidity strength of benzoic acid is related to inductive effect inside the molecule, which is practically observable effect of the move of charge through atoms in benzoic acid molecule resulting in a continual state of bond polarization [11]. Over $30^{\circ} \mathrm{C}$, benzoic acid molecule behaves less acidic due to the decrease in the effect of electron releasing group inside the benzoic acid molecule on the acidic hydrogen as observed from its numerical values of $p K a$ at different temperatures.

\section{Conclusion}

The observed solubility and $\mathrm{pH}$ values of benzoic acid in water related inversely with $\mathrm{NaCl}$ concentrations. The calculated apparent dissociation constant $(K c)$ is directly related. No regular correlation is between the thermodynamic dissociation constant of benzoic acid and temperatures between $16^{\circ} \mathrm{C}$ and $41^{\circ} \mathrm{C}$. The dissociation process of benzoic acid molecule around $25^{\circ} \mathrm{C}$ is non-spontaneous.

\section{Acknowledgements}

The help in the arrangement of equations by Prof. Khalid Tawarah from Yarmouk University-Irbid is highly appreciated.

\section{References}

[1] Smetana, A.J. and Popov, A.I. (1979) On the Influence of Ionic Strength on Equilibrium Constant of Ion- Molecule Reaction. Journal of Chemical Thermodynamics, 11, 1145-1149. http://dx.doi.org/10.1016/0021-9614(79)90106-X

[2] Tawarah, K.M. and Khouri, S.J. (2000) Determination of the Stability and Stoichiometry of p-Methyl Red Inclusion Complexes with $\gamma$-Cyclodextrin. Dyes and Pigments, 45, 229-233. http://dx.doi.org/10.1016/S0143-7208(00)00024-3

[3] Sergeeva, V.F. (1965) Salting-Out and Salting-In of Non-Electrolytes. Russian Chemical Reviews, 34, 309-318. http://dx.doi.org/10.1070/RC1965v034n04ABEH001446 
[4] Albert, A. and Serjeant, E.P. (1984) The Determination of Ionization Constants. 3rd Eddition, Chapman and Hall, London. http://dx.doi.org/10.1007/978-94-009-5548-6

[5] Atkins, P. and de Paula, J. (2010) Physical Chemistry. 9th Eddition, W.H. Freeman and Company, New York.

[6] Vogel, A. (1989) Text-Book of Quantitative Chemical Analysis. 5th Edition, Longman, Harlow.

[7] Sarmini, K. and Kenndler, E. (1998) Capillary Zone Electrophoresis in Mixed Aqueous-Organic Media: Effect of Organic Solvents on Actual Ionic Mobilities, Acidity Constants and Separation Selectivity of Substituted Aromatic Acids II. Ethanol. Journal of Chromatography A, 811, 201-209. http://dx.doi.org/10.1016/S0021-9673(98)00256-8

[8] Christensen, J.J., Hensen, L.D. and Izaft, R.M. (1976) Handbook of Ionization Constants. Wiley Interscience, New York.

[9] Bosch, E., Bou, P., Allemann, H. and Rosés, M. (1996) Retention of Ionizable Compounds on HPLC. pH Scale in Methanol-Water and the $\mathrm{pK}$ and $\mathrm{pH}$ Values of Buffers. Analytical Chemistry, 68, 3651-3657. http://dx.doi.org/10.1021/ac960104l

[10] Cleveland, J.A., Benko Jr, M.H., Gluck, S.J. and Walbroehl, Y.M. (1993) Automated pK $K_{\mathrm{a}}$ Determination at Low Solute Concentrations by Capillary Electrophoresis. Journal of Chromatography A, 652, 301-308. http://dx.doi.org/10.1016/0021-9673(93)83247-P

[11] Solomons, G.T. and Fryhle, C.B. (2011) Organic Chemistry. 10th Edition, John Wiley \& Sons, Inc., Hoboken. 